\title{
La orientación educativa como campo de intervención: origen, sentidos y perspectivas
}

\author{
The educational orientation as an intervention field: origins, meanings and \\ perspectives
}

César Martín Barletta cesarbarletta@gmail.com

Facultad de Humanidades y Ciencias de la Educación. Universidad Nacional de La Plata, Argentina

Recepción: 05 Mayo 2020

Aprobación: 12 Marzo 2021

Publicación: 01 Abril 2021

Cita sugerida: Barletta, C. M. (2020). La orientación educativa como campo de intervención: origen, sentidos y perspectivas. Archivos de Ciencias de la Educación, 14(18), e087. https://doi.org/10.24215/23468866e087

\begin{abstract}
Resumen: Este artículo intenta contribuir con los estudios acerca de los orígenes de la orientación educativa en Argentina y su contexto de surgimiento, justificación e implicación como un saber especializado, diferenciado y como práctica profesional. Para ello realizaremos la presentación, análisis y descripción de un conjunto de discursos que, en las dos primeras décadas del siglo XX, comenzaron a delimitar un vasto campo de intervenciones atravesado por un conjunto de prácticas, saberes y posiciones profesionales heterogéneas. Definiremos a la orientación educativa como campo de intervención, exploraremos los orígenes del término y los primeros acuerdos conceptuales que se realizan en Europa y Estados Unidos con la intención de diferenciar los alcances de la orientación en las escuelas y fuera de ellas.
\end{abstract}

Palabras clave: Orientación educativa, Orientación profesional, Campo de intervención, Escuela.

\begin{abstract}
This article aims at contributing to the studies about the origins of educacional orientation in Argentina and the context of its introduction, justification and implications as a specialized knowledge, diferentiated and as a professional practice. For that we will introduce, analyze and depict a set of discourses that started to delimitate a vast intervention field related to a set of practices, knowledges and heterogeneous professional positions, starting from the first two decades of the twentieth century. We will define the educacional orientation as a field of intervention exploring the inception of the term, and the first conceptual agreements produced both in Europe and the United States that intended to distinguish the orientation's scopes inside and outside the schools.
\end{abstract}

Keywords: Educational orientation, Professional orientation, Intervention field, School.

\section{La Orientación Educativa como CAMPo de intervención}

Entendemos a la Orientación Educativa como un campo de intervención dirigido a la creación de culturas institucionales, proyectos y prácticas inclusivas, tanto curriculares como de enseñanza, realizadas por un equipo de profesionales interdisciplinario y dirigidas a la totalidad de los estudiantes en el espacio escolar. 
Se trata de un trabajo intersectorial que priorice y revalorice la creación de vínculos y grupos capaces de tejer redes sociales que propicien o refuercen relaciones de colaboración y convivencia democrática entre los agentes y sujetos educativos, así como con la comunidad, desde una perspectiva de derechos humanos y con una fuerte impronta territorial.

Según Bourdieu, un campo es un espacio de puja entre agentes que intentan hegemonizarlo.

La estructura del campo es un estado de la relación de fuerzas entre los agentes o las instituciones que intervienen en la lucha o, si ustedes prefieren, de la distribución del capital específico que ha sido acumulado durante luchas anteriores y que orienta las estrategias ulteriores (...) Para que funcione un campo es necesario que haya algo en juego y gente dispuesta a jugar, que esté dotada de los habitus que implican el conocimiento y reconocimiento de las leyes inmanentes al juego, de lo que está en juego, etc. (Bourdieu, 1990, pp. 135-136).

Se ha concebido a la orientación educativa "como campo, y no sólo como disciplina, porque va más allá de un cúmulo de conocimientos y traspasa los límites de la pedagogía y la psicología; hacia espacios relacionados con la sociología, la economía, la filosofía y la historia" (Pacheco, 2013, p. 26). La noción de campo nos ayuda a interrogarnos sobre diferentes preguntas que refieren al proceso histórico de su construcción, tiene un cúmulo de conceptos teóricos y es una estrategia de intervención en las escuelas de diferentes niveles (...) Así, el concepto de campo de la Orientación Educativa permite tener esta panorámica de su surgimiento y de los debates, en lugar de tomar sus concepciones como productos acabados, tendrán una referencia obligada a su construcción (Pacheco, 2013, p. 26).

De esta manera, la búsqueda de los diferentes posicionamientos históricos que han legitimado ciertas formas de pensar, actuar y hacer orientación educativa se presentan como la posibilidad de realizar una revisión crítica de las tendencias hegemónicas dentro del campo y establecer un conjunto de desafíos para su restructuración, así como para la creación de estrategias y relaciones que pongan énfasis en los procesos de inclusión escolar y social y no en los aspectos de diagnóstico, información y ajuste (tendencia conservadora industrial) o en funciones de intervenciones para la toma de decisiones, el autoconocimiento y el desarrollo de la autoestima (tendencia neoliberal posindustrial) también con fines de adaptación de una forma más dinámica y con métodos y técnicas más sutiles.

En este sentido, la orientación educativa se presenta no sólo como campo de intervención sino también como campo problemático. Un campo problemático es definido por Zemelman como "campo de fenómenos que contiene diversas modalidades de concreción, dado que los distintos procesos que la conforman se articulan según sus particularidades espacio-temporales y dinamismos estructurales y coyunturales" (Zemelman y Martínez, 1987, p. 57).

A continuación, realizaremos una lectura de algunos discursos que dieron origen al campo de intervención en orientación educativa en Argentina a inicios del siglo XX. Específicamente nos referiremos a las cuatro primeras décadas del siglo pasado debido a que no estamos sólo frente a la conformación de un campo de intervención específico, sino que, además, es el momento fundacional de la Argentina liberal-oligárquica.

Los cambios acontecidos en la nación en definiciones sobre la educación, el lugar de los niños y adolescentes, la ciudadanía, el surgimiento de saberes especializados de control, cuidado, selección y clasificación, así como la consolidación de una moral burguesa y la división social del trabajo, son temáticas cruciales para el entendimiento del tema elegido y hacen a su relevancia como estudio de las ideas y prácticas educativas.

Por último, es importante resaltar que conocer y analizar esos discursos noveles acerca de los alcances de la orientación posibilitará reconocer un conjunto de marcas identitarias que continúan vigentes, y otras que han sido resignificadas en relación con la medicalización, patologización y judicialización de las infancias y juventudes en la actualidad. 


\section{ORIGEN DE LA ORIENTACIÓN EDUCATIVA: ASPECTOS VOCACIONALES Y PSICOPEDAGÓGICOS}

Diferentes autores (Rodríguez Moreno, 1999; Sebastián Ramos, 2003; Gavilán, 2006; Lledó Becerra, 2007) señalan que las prácticas orientadoras surgieron a inicios del siglo $\mathrm{XX}$ tanto dentro del ámbito escolar, como por fuera de él, en respuesta a un conjunto de preocupaciones socioeducativas. Las más apremiantes tenían que ver con el trabajo, guía y asesoramiento sobre los aspectos vocacionales de los sujetos al concluir la escolarización y con la creación de técnicas e instrumentos de exploración psicopedagógicas acerca de los intereses, la inteligencia, memoria y aptitudes de niños y jóvenes. La finalidad de las prácticas orientadoras se relacionaba con la construcción de un saber sobre las infancias y juventudes que, amparado en las bases científicas de la época, contribuyera a ordenar el espacio escolar y social distribuyendo lugares, instituciones y oficios, y a atender las demandas provenientes del mundo educativo y del mundo del trabajo.

Los primeros años del siglo XX fueron, además, el escenario propicio para el desarrollo de las profesiones de ayuda o terapéuticas en donde la orientación educativa se ubica como una estrategia o instrumento de consulta o como facilitación dentro de los marcos del sistema de educación formal:

De todos modos, las personas que se interesaban por los problemas profesionales producto de los cambios sociales e industriales de principio de siglo eran reformadores sociales y profesionales de la ayuda. Tanto en Europa como en Norteamérica se aceptó que la orientación era una función básica de la educación pública y que debía estar centrada en la escuela (Rodríguez Moreno, 1998, p. 13).

El surgimiento de estas profesiones responde a lo que Foucault (2007) denomino función psi:

La función psi se convierte a la vez en el discurso y el control de todos los sistemas disciplinarios. Es el discurso y la introducción de todos los esquemas de individualización, normalización y sujeción de los individuos dentro de los sistemas disciplinarios (Foucault, 2007, p. 111).

Sumado a ello, encontramos distintas tendencias intelectuales de la época que contribuyeron a la incorporación de la investigación científica de carácter experimental en la educación. En este sentido hay que mencionar al positivismo que, como corriente filosófica, impulsó el empleo de los procesos estadísticos en la investigación educativa; a la teoría de la evolución, que permitía establecer los estudios del niño y de su desarrollo, pero que también propició el surgimiento de distintas ciencias auxiliares como la antropometría y la cefalometría, en las que las mediciones obtenían un lugar predominante; y a la psicología experimental, que comenzaba a medir la inteligencia mediante la creación y utilización de diversos test psicopedagógicos (Sebastián Ramos, 2003; Southwell, 2003; Haidar, 2013).

En el marco de este contexto de producción social, económica e intelectual es que aparece la noción de talento como eje de los procesos de individualización. Se buscaba encontrar las peculiaridades de cada talento, así como los condicionamientos y posibilidades presentes o ausentes en cada sujeto. $\mathrm{Al}$ respecto, pueden rastrearse algunos antecedentes. En Francia, en 1842, se edita la Guide pour le choix dun etat, a modo de diccionario de profesiones. Esta fue la "primera obra de su género, compuesta por un conjunto de monografías profesionales en la que se daba gran importancia a las aptitudes personales de los sujetos y a la preparación profesional" (Rodriguez Moreno, 1998, p. 13). En 1869 Galton publica Hereditary Genius, obra en la que afronta el tema de las diferencias individuales. Primer promotor en la utilización de los test o del movimiento psicométrico, su línea de investigación tiene continuación en los estudios sobre la inteligencia de Spearman (Inglaterra). Se publica también la Psychologie Individuelle (1895), de Binet y Henri. En Alemania, W. Wundt (1897) abre el primer laboratorio de Psicología Experimental orientado al estudio de las diferencias de los sujetos desde un punto de vista biomédico, y ya en 1902 comienza a funcionar una primera oficina de orientación profesional creada por sindicatos y cámaras de comercio. En 1905, Binet y Simon realizan la primera escala de inteligencia e introducen el concepto de edad mental.

Para los orientadores, estos nuevos expertos que comenzaban a ingresar al espacio escolar, la utilización de los preceptos psicométricos "representó un modo de hacer más científico su trabajo, ya que supuestamente 
estas pruebas permitían medir y predecir tanto el éxito académico como laboral, con instrumentos supuestamente rigurosos, confiables y válidos" (Santana Vega, 2003, p. 63).

En Estados Unidos la orientación surge como tarea extraescolar ligada a tareas de selección e inserción ocupacional. A estos requerimientos intentó dar respuesta Frank Parson (1854-1908). Ingeniero transformado en asistente social, abrió una residencia en Boston para jóvenes trabajadores o en búsqueda de empleo, el "Vocational Bureau" (oficina vocacional). Parson fue, además, quien acuño el término vocational guidance (orientación vocacional) en su libro Choosing and Vocation (1909), en donde describe su método de tratamiento a los jóvenes y señala la necesidad de que la Orientación Vocacional formara parte de los programas de los centros públicos y fuera atendida por expertos.

El papel de la orientación, según este autor, debería ser el conseguir que cada individuo encontrase el trabajo más adecuado, lo que sería ventajoso tanto para el individuo como para la sociedad. Bajo el lema "cada hombre en una profesión, una profesión para cada hombre", este modelo se ha denominado de "rasgos y factores" o "teoría de la adecuación" entre individuo y profesión. Se basaba en el diagnóstico del sujeto y la orientación se concibe como un hecho puntual y directivo con apenas protagonismo de la persona orientada. Con la muerte de Parsons en 1908, Meyer Bloomfield asume la dirección del Vocational Bureau y lo integra a la Universidad de Harvard. Él mismo impartió el primer curso de orientación vocacional para universitarios en 1911. También ese mismo año publicó The vocational Guidance of Youth, obra en la que expone la importancia de que los alumnos encuentren ayuda en los momentos tan decisivos en la vida como el paso de la escuela al trabajo. Encontramos otros antecedentes en los aportes de George Merrill, quien comienza en 1895 con el primer intento sistemático de servicios de orientación en la Escuela de Artes Mecánicas de San Francisco. Merrill proponía a los alumnos la realización de experiencias exploratorias en cada uno de los oficios que se enseñaban en la escuela, al mismo tiempo que atendía a los antiguos alumnos y procuraba su colocación en caso de necesidad. J. Davis (1898) inicia sus actividades orientadoras con los alumnos de la Central Hight School en Detroit, Michigan, trabajando en la integración curricular de la orientación dentro de la escuela y realizando tareas de asesoramiento a los estudiantes sobre aspectos vocacionales y problemas educativos. De la misma manera W. Harper, primer presidente de la Universidad de Chicago, en su discurso de 1898 "Scientific Study of the Student", resalta la necesidad de individualizar la enseñanza y al mismo tiempo plantea la llegada de expertos en orientación para los estudiantes universitarios de los primeros años.

Diferentes autores (Sebastián Ramos, 2003; Santana Vega, 2003) plantean que, en forma simultánea a los desarrollos anglosajones aparece el movimiento de orientación europeo, estrechamente vinculado a factores económicos y sociales, motivado por el convencimiento de que la orientación podría ser el medio de corregir las desigualdades provocadas por el origen familiar. Así, la orientación se enfocó en la nueva clase trabajadora y trataba de conectar a la escuela con el mundo del trabajo: el movimiento europeo tenía una preocupación específica por la relación entre trabajo y escuela. Para lograr esta conexión se desarrollaron intervenciones junto a escuelas de aprendices, talleres de oficios, cámaras de comercio, etc., entidades que actuarán como nexo para el desarrollo de las actividades extraescolares. Asimismo, se hizo un gran esfuerzo en pos de la medición de las aptitudes de los futuros trabajadores a partir de los aportes de la psicología aplicada y la psicología diferencial. Los estudios se llevaron a cabo en el campo de la psicofisiología y la organización científica del trabajo con el objetivo de lograr la oportuna coordinación entre la estructura ocupacional y la mano de obra, mediante mecanismos de orientación y/o selección.

\section{Los discursos sobre la orientación eduCATIVA y PRofesional en Argentina}

En Argentina, a principios del siglo XX, una serie de cuestiones que posteriormente han entrado en la órbita de lo que actualmente se denomina orientación educativa comenzaron a despertar el interés de quienes se dedicaban a la educación, a la incipiente psicología y a la administración y planificación estatal. La recepción de estas innovaciones por parte de quienes se dedicaban a las tareas pedagógicas (maestros, 
profesores o médicos), sensibilizados por los conocimientos de la psicología aplicados a la educación, vino de la mano de la corriente paidológica que propició el surgimiento de movimientos como el de la Escuela Nueva, dándose importancia a la diversidad de los estudiantes, a la orientación escolar y profesional, a las condiciones de escolarización (higiene escolar) y sobre todo a la propia infancia (Southwell, 2003).

Esta situación permitió el desarrollo de la educación compensadora para todos aquellos "niños deficientes" o para las "infancias anormales", situaciones que no fueron ajenas a la conformación de los primeros discursos sobre orientación educativa que se configuraban en torno a dos grandes ejes: la definición, clasificación e intervención médica o psicopedagógica de las infancias anormales o débiles y la relación entre mercado laboral y la educación.

\section{La orientación educativa: discursos, sentidos y orígenes en Argentina}

El disciplinamiento y normalización de las infancias y de los jóvenes en las escuelas constituyó un amplio campo de encuentro entre disciplinas y profesionales que posibilitaron la construcción del sujeto problemático, así como los mecanismos y dispositivos necesarios para su detección, tratamiento y segregación. En ese encuentro, el saber psiquiátrico se constituyó como aquel que detenta "la verdad" sobre las infancias problemáticas y cumplió una función de asesorar, guiar y complementar al saber pedagógico.

Confluyen, además, un conjunto de discursos provenientes de la psicología, la medicina y el derecho en la construcción de un nuevo lenguaje experto definido por la pericia. La "pericia" se consolida como el estudio y la búsqueda de un conjunto de indicios y elementos biográficos en los niños y jóvenes que podría predecir y regular tanto la conducta social como el éxito o fracaso escolar. Se inscribe como "el saber experto" para delimitar un conjunto de prácticas divisorias, tanto de los saberes como de los sujetos e instituciones. Al decir de Valerie Harwood, "las practicas divisorias son un medio eficiente para facilitar acciones sobre las acciones de los otros debido a que su función es identificar y aislar al individuo. Estas prácticas producen el efecto de objetivar al sujeto" (2009, p. 108). Para objetivar al niño era necesario nombrarlo, delimitarlo, conocerlo y reconocerlo en la especificidad de un cuerpo que necesitaba ser protegido, estudiado y controlado. De esta manera, se consolidaron fronteras que dividieron las vidas de las infancias y juventudes mediante el establecimiento de un orden clasificador primigenio entre niños y menores.

El concepto de menor es emblemático de esta confluencia de sentidos que terminan constituyendo una zona opaca. Presente en las teorías del derecho y en la filosofía desde los comienzos de ambas construcciones teóricas, la noción da a ver y pone de manifiesto una significación móvil que transforma al menor de edad, categoría jurídica que concierne a todos los niños con independencia de su origen, en un menor, noción que carga con las representaciones de una infancia adjetivada, qué se ha podido leer, a lo largo de los tiempos, bajo distintos nombres que responden a un orden clasificador. (Frigerio, 2008, p. 10)

El proceso clasificador de niños y jóvenes determinó prácticas diferenciales en las instituciones, en las posiciones profesionales y en los vínculos intergeneracionales. Con relación a los saberes, las prácticas divisorias encuentran en su especialización y jerarquización la posibilidad de un sustento teóricocientífico, basado en diagnósticos y adjetivaciones sobre niños y jóvenes. Dentro del lenguaje escolar, el diagnóstico definido como el estudio de los aspectos físicos, psíquicos y pedagógicos, posibilitaría detectar "anormalidades" o "deficiencias" en los sujetos para su posterior tratamiento, reeducación o segregación.

Mediante diferentes instrumentos de observación, registro y documentación, se pondrían de relieve las diferentes capacidades y aptitudes a través de la medición y clasificación de las inteligencias. La función diagnóstica en la escuela basó sus acciones en los desarrollos del movimiento psicométrico, la utilización de test, los estudios sobre la memoria y las escalas de inteligencia en donde se introduce el concepto de edad mental, lo que aseguraría un conocimiento exacto y verdadero, ya que 
gracias a los progresos de la psicología experimental contamos hoy con métodos objetivos para la determinación de las aptitudes. Mediante ciertas pruebas "tess", es posible determinar el grado de inteligencia de los sujetos, su memoria, su atención, su habilidad mental, etc. (Fingerman, 1926, p. 43).

Asimismo, "la clasificación de los especialistas del dominio médico-pedagógico converge pues con la de la escuela, reforzando, mediante la aportación de un aparato científico de medición, la ideología de los dones naturales" (Lunardi, 2002 p. 142).

En relación con los sujetos, las prácticas divisorias nos asisten en la comprensión de dos situaciones. En primer lugar, aquellas destinadas a la división de las infancias y juventudes como "normales y anormales"; en segundo lugar, las prácticas destinadas a compartimentar las tareas de los profesionales de la educación para la llegada a las instituciones de un nuevo grupo de expertos.

El proceso de constitución de las infancias como anormales o problemáticas comenzaba con el estudio de las condiciones físicas de los estudiantes o, en caso de ser muy evidente, con la confirmación de un diagnóstico predefinido debido a que se suponía que se podían detectar problemas internos por medio de rasgos fisiológicos. Con esa intención es que se planteaba la inspección,

no sólo a los sanos, sino también a los enfermos. Por eso llamamos a orientar sobre todo a los tuberculosos... habrá que distinguir entre los sujetos que tienen aptitudes más bien manuales y los que están dotados preferentemente de condiciones intelectuales (Laufer, 1923, p. 126).

Las características físicas debían acompañarse por el estudio de las condiciones pedagógicas, familiares y sociales de los niños. Para ello es que se establecen nuevas divisiones dentro del trabajo escolar y se crea la necesidad de apoyar y guiar el trabajo pedagógico a partir de la incorporación de nuevos expertos. El médico escolar, los maestros visitadores y los profesionales con conocimientos psicológicos harán su ingreso en la escuela, y estos expertos se distinguen de sus colegas pedagógicos por poseer conocimientos sobre la salud mental del niño. Esa "sabiduría" tuvo como consecuencia inmediata el establecimiento de diferentes grados de conocimiento, donde el maestro aparece como aquel que detenta un mayor "grado de desconocimiento" y por lo tanto se convierte en el individuo que necesita el consejo y guía del experto.

En esta nueva división del trabajo, corresponde al maestro detectar e informar las desviaciones que detecta en sus clases. Para ello deberá llevar un registro lo más detallado posible, que sea útil para que los orientadores estén lo "suficientemente documentados" y, en caso de ser necesario, "corregir o desviar, intelectualmente, al niño" (Foulégne, 1928, p. 221). Además, se define como indispensable la introducción de dos dispositivos que colaboran con la información de los exámenes y registros pedagógicos. Por un lado, la conformación de libretas sanitarias con información individual, familiar y social, donde se

proporcionan datos precisos sobre los antecedentes patológicos, la historia física de cada alumno, su agudeza visual y auditiva, el estado de sus órganos, las perturbaciones o enfermedades de que padece o que pueden sobrevenir en el curso del tiempo que pasará en la escuela (Laufer, 1923, p. 127);

por otro lado, la realización de visitas a los hogares de les estudiantes con la intención de confeccionar libretas sociales, que ya no realizarán los médicos escolares sino un grupo de enfermeras que brindan información sobre la higiene en las habitaciones o casas de los individuos.

A partir de esta incorporación de expertos y de las funciones que desarrollaron es que podemos comenzar a delimitar un conjunto de rasgos que luego consolidarían el lugar de la orientación educativa en las escuelas como una figura experta en la guía, el asesoramiento y la ayuda a maestros, y en la realización de diagnósticos individuales y vocacionales a los estudiantes. Se enfatiza un modelo jerárquico de separación de funciones de tal forma que el orientador aparece como el técnico diagnosticador, el maestro como el usuario del conocimiento elaborado por el experto y el alumno es el agente de la intervención orientadora.

En relación con las prácticas divisorias de las instituciones, las repercusiones de estas evaluaciones y la posterior categorización de niños y jóvenes incluían la toma de decisión sobre su permanencia en la escuela, aunque en aulas separadas, o su traslado hacia las escuelas especiales. Una vez que se han identificado, 
conviene, sin duda, al maestro conocer las reglas médicas apropiadas al tratamiento de los deficientes; pero es de suponer que la educación especial de estos desgraciados no se hará sino en establecimientos en que el maestro comparta sus tareas con el médico mismo. Nos limitaremos, pues, a la parte más propiamente pedagógica (Ingenieros, 1906, p. 197).

En este sentido se instauraba la posibilidad de establecer los límites de la inclusión y la exclusión educativa a partir de la conformación dual del sistema normalidad/anormalidad como principio tautológico con base profundamente moral. Las infancias se dividen entre aquellas que asistirán a escuelas normales y aquellas que se incorporarán a sistemas institucionales educativos paralelos: el sistema de educación especial y el sistema de tutela a menores. Diagnósticos, clasificaciones y segregaciones que marcaban procesos de socialización distintos y trayectorias signadas por la capacidad estructurante de las instituciones o la institucionalización de vidas dañadas.

\section{Retrasados y delincuentes: los sujetos de la orientación}

Hubo una época, hace unos años, en que se podía aclarar todo con simplemente decir "débil mental". Era una expresión maravillosa porque parecía resolver muchos problemas. Si un niño robaba, era un débil mental; si se cometía un asesinato, el asesino era débil mental; si un niño no quería ir a la escuela, debía ser débil mental; y si iba a la escuela y exhibía comportamientos desagradables, seguro que era débil mental. El retraso mental era una explicación suficiente para todos estos problemas (Wile 1926, citado en Harwood, 2009, p. 112).

La formulación científica en la construcción de la anormalidad y de los sujetos problemáticos estuvo en un primer momento abocada a la clarificación y distinción de los distintos niveles de retraso, así como a las condiciones físicas y sociológicas que eran su causa. Primaba la construcción de una terminología precisa que permitiera la realización y respaldo de los diagnósticos, y para ello se recurrió a los saberes expertos provenientes de la psicología y la psiquiatría.

Esta situación permitió el establecimiento de fronteras y diferenciaciones entre dos grandes nuevas figuras dentro del espacio escolar: el "retrasado escolar" y el "niño peligroso" o "delincuente". Comienza de este modo una práctica institucional de división e identificación de las infancias como problemática, peligrosa o en peligro. Las prácticas de división han sido un medio particularmente eficiente para clasificar y aislar al individuo categorizado como problemático, para planificar itinerarios alternos de escolarización y para convertir su comportamiento como especial.

Es en la figura del "retrasado escolar" en donde podemos sintetizar una serie de injerencias profesionales que ingresarían en la órbita de la orientación educativa. Es una labor referida a evidenciar las diferencias individuales basadas en rendimientos, historia familiar y el estado y características físicas. Las áreas más desarrolladas para esas finalidades se relacionaron con la evaluación y organización educativa en grados de acuerdo con las capacidades mentales de los estudiantes, el diagnóstico de retardos escolares, la prescripción de tratamientos médico-pedagógicos y la orientación vocacional-laboral.

El "retrasado escolar" es quien no adecua sus aprendizajes respecto de una temporalidad preasignada en años escolares, quien no cumple con los aprendizajes pautados para cada año escolar. Esta definición se vincula de hecho a la de deficiencia, representada por el niño o joven "deficiente"; es decir, se trata de hacer de un problema pedagógico una característica propia del sujeto. La función es objetivar al sujeto para estudiarlo, clasificarlo y jerarquizarlo.

...en la educación de niños normales tenemos una base para el desarrollo mental según la edad del educando, base que nos permite fijar el término medio de educación que le corresponde, mientras que en los anormales el grado de atraso es demasiado variable y en la mayoría de los casos en ninguna relación con la edad del niño (Gaye, 1924, p. 28).

Si la figura del retrasado condensa una serie de características escolares para su definición y tratamiento, la figura del niño peligroso o en peligro aglutina no solo aquella referencia sino también una preocupación por el mantenimiento del orden social. La noción de retrasado se asocia a la de peligrosidad. "Cada retardado es 
un criminal en potencia. De diez a veinte por ciento de todos los ocupantes de las instituciones penales son anormales mentales" (Nelson, 1920, p. 162). Se trata de una peligrosidad individual y social que los maestros y médicos dentro de las escuelas deben poder prever, detectar e informar para la separación del sujeto peligroso y para su tratamiento y/o confinamiento en sistemas educativos y/o tutelares paralelos.

Al emprender el tratamiento y la educación de un niño que se encuentre en condiciones psíquicas anormales, el objeto del médico y del educador tenderá a poner al sujeto en condiciones que le hagan cada vez menos indispensable la ayuda ajena y le permitan, en lo posible, bastarse a sí mismo. Procurará, además, corregir las tendencias e impedir los actos que puedan hacerlo peligroso para sí mismo o para la sociedad (Ingenieros, 1906, pp. 198-199).

\section{La orientación profesional: educación y trabajo}

La intervención en orientación profesional se delimita en los márgenes de una tríada conformada por el trabajo, la salud y la educación. Es presentada como una acción metódica y predictiva con la intención de influir, asesorar y ayudar a los jóvenes en sus trayectos laborales a partir de un análisis sistemático y experto de las características individuales, familiares, escolares y sociales.

"La orientación profesional es por eso un problema económico, ético, social y psicológico" (Fingerman, 1926, p. 40), que debe tratarse en forma científica para ayudar a los adolescentes a elegir y decidir una carrera para toda la vida y contrarrestar el problema de cambios de oficios o profesiones, no solo durante la vida de trabajador sino también para aquellos que eligen estudios universitarios y luego cambian de carrera o no la finalizan.

Ya nadie discute la utilidad de la orientación profesional. Todos reconocen que su objeto es de aconsejar al niño, generalmente al salir de la escuela primaria, una profesión que responda a sus gustos e intereses, a sus conocimientos y aptitudes diversas, teniendo en cuenta la situación de la familia y el estado del mercado de trabajo (Foulégne, 1928, p. 219).

En 1923, Renato Laufer (médico e inspector de escuelas) escribe "Las bases médico-sociales de la orientación profesional”, donde plantea un interrogante: “¿Se basa actualmente la orientación profesional en el estado de salud y las capacidades reales?". La respuesta es que la mayor parte de los “jóvenes adoptan un oficio por casualidad, por ocasión, por imitación o impresión, bajo la influencia de sus padres, de un amigo, de conocidos, y más a menudo, por el señuelo de un salario inmediato" (Laufer, 1923, p. 125).

La búsqueda de un método científico de ayuda que oriente a niños y jóvenes a encontrar su lugar en la sociedad, de acuerdo con sus capacidades intelectuales y condiciones de salud, nos permite encauzar estos discursos dentro de las preocupaciones eugenésicas del momento y del armado de distintos dispositivos para el gobierno de la vida por parte del Estado. Se plantea que la orientación profesional "restringirá el número de los amargados y los que se sienten fuera de su clase”. (Laufer, 1923, p. 125). Así entendido, el problema de la orientación profesional forma parte de los temas del higienismo social:

dadas las aptitudes del interesado, sus inclinaciones, sus cualidades morales, ¿cuál es el oficio que tiene más probabilidades de ejercer, salvaguardando su salud, sus facultades de producción y aun favoreciendo su desarrollo, es decir, con el mejor provecho de sus intereses personales y del interés general? Se ve que, para nosotros, la orientación es un capítulo importante de la higiene social (Laufer, 1923, p. 126).

\section{La formación de expertos para la OP}

La orientación profesional como acción metódica y predictiva necesitaba de la formación de expertos para el trabajo acerca de los aspectos vocacionales de los niños y jóvenes, así como del conocimiento de las profesiones y oficios que se ajustan a sus perfiles. Se debería trabajar sobre 
la vocación, preguntando al muchacho sobre la profesión elegida por él; inquirimos sobre sus gustos, sus ocupaciones dentro y fuera de la escuela. Examinamos sus trabajos escolares, sus dibujos, etc... y sus clasificaciones. Luego comprobamos por el examen fisiológico y psicológico si el sujeto posee realmente las aptitudes requeridas para el oficio elegido. A veces basta un examen físico o fisiológico para mostrar deficiencias insalvables en los sujetos para el oficio preferido (Fingerman, 1926, p. 47).

Ese trabajo sobre los aspectos vocacionales debía acompañarse de un conocimiento de la oferta de oficios. Hay una valorización del trabajo manual, de los oficios y ocupaciones. Se plantea

la importancia que conviene dar a un conocimiento tan exacto como posible del estado del mercado de trabajo (oficios con escasez y exceso de oferta, oficios en vías de desaparición y gremios en estado de evolución: lo que explica la colaboración de las asociaciones gremiales, patronales y obreras) (Foulégne, 1928, p. 220).

Los maestros no pueden ser orientadores, pero su función debe ser la de un preorientador que debe

formar en el niño una mentalidad profesional (...) exaltar en él el amor al trabajo en general y el respeto del trabajo manual en particular; significa sugerirle que cualquier oficio vale tanto como otro: que no existen oficios "toscos", que solamente hay gente "tosca" (Foulégne, 1928, pp. 220-221).

La República Argentina fue el primer país de América del Sur que contó con un instituto de orientación profesional dotado de un laboratorio psicotécnico. La iniciativa de su formación se originó en el "Congreso del Trabajo" (Rosario, 1923) que abogó por la creación de un "Instituto Central de Orientación Profesional”. Creado en 1925 por decreto del Poder Ejecutivo Nacional, su objetivo sería el de "examinar a todos los jóvenes próximos a finalizar la escuela, estableciendo un diagnóstico sobre la base de la idoneidad corporal, la vocación, la situación económica tanto del joven como de su familia y la situación del mercado de trabajo" (Jesinghaus, 1924, s/p).

La creación del instituto respondió a dos problemáticas que se plantean a inicios del siglo XX: la inserción de los trabajadores en la escala productiva y la adecuada selección y orientación para aquel fin. Al respecto, Gregorio Fingerman, Profesor del Instituto de Psicotécnica y de Orientación Profesional, plantea que

lo que se hace necesario, entonces, es preparar al alumno en vista de las futuras profesiones posibles y orientarlo cuando está por abandonar las aulas. No se le puede dejar librado al azar y entregarlo a la lucha en condiciones desventajosas. El niño se ignora a sí mismo, no conoce sus capacidades reales. Los padres, por lo general gente del pueblo, tampoco son más hábiles para encaminar a sus hijos en la vida profesional. Y sin embargo la elección de una carrera o de un oficio es de capital importancia porque decide para toda la vida (Fingerman, 1926, pp. 39-40).

\section{A MODO DE CIERRE}

Este artículo se limitó a explorar aspectos sociales, históricos y pedagógicos que contribuyeran a pensar la génesis y desarrollo del campo de intervención de la orientación educativa y de la orientación profesional en Argentina. La noción de campo nos ha permitido reconocer los comienzos, desarrollos y consolidación de la orientación educativa a la luz del conocimiento y comprensión de las fuerzas que lo han atravesado mediante la proliferación de discursos acerca de los tiempos, momentos y lugares de educabilidad de los sujetos y de su inserción sociolaboral en el marco de políticas direccionadas a ordenar, educar y orientar a la sociedad, sujetas a cuestiones morales, sexuales, educativas o productivas. En este sentido, el reconocer las marcas de origen de la orientación educativa permite que, pasado un siglo desde los primeros desarrollos, hoy entendamos que se trata de un campo de intervención dirigido a la creación de culturas institucionales, proyectos y prácticas inclusivas, tanto curriculares como de enseñanza, realizadas por un equipo de profesionales interdisciplinario y dirigidas a la totalidad de los estudiantes en el espacio escolar. Este campo de intervención en la actualidad ha desarrollado líneas de actuación profesional que resumen nuevas miradas y conceptualizaciones sobre los procesos de orientación: tareas de asesoramiento, acompañamiento de trayectorias, el lugar predominante 
de la enseñanza y la búsqueda de apoyos pedagógicos para estudiantes y docentes resumen el camino actual hacia una escuela inclusiva.

\section{REFERENCIAS BIBLIOGRÁFICAS}

Bourdieu, P. (1990). Algunas propiedades de los campos. En Sociología y Cultura. México: Grijalbo

Fingerman, G. (1926). La orientación profesional y la escuela. Monitor de la Educación Común. Buenos Aires. Consejo Nacional de Educación, 37-49

Foucault, M. (2007). El poder psiquiátrico. Buenos Aires: Fondo de Cultura Económica

Foulégne, J. (1928). La orientación profesional en Francia. Monitor de la Educación Común. Buenos Aires. Consejo Nacional de Educación, 47 (664), 219-226

Frigerio, G. (2008). La división de las infancias. Ensayo sobre la enigmática pulsión antiacóntica. Buenos Aires: Del estante editorial

Gavilán, M. (2006). La transformación de la Orientación Vocacional: hacia un nuevo paradigma. Rosario: Novedades Educativas

Gaye, J. S. d. P. (1924). Las escuelas de anormales. Monitor de la Educación Común. Buenos Aires. Consejo Nacional de Educación, 28-30

Haidar, V. (2013). La orientación profesional en la Argentina (1920-1955): un ejercicio de historia del presente. Revista Mundos do Trabalho, 5 (9), 331-358

Harwood, V. (2009). El diagnóstico de los niños y adolescentes problemáticos. Una crítica a los discursos sobre los trastornos de conducta. Madrid: Morata.

Ingenieros, J. 1. (1906). La educación de los niños deficientes. Monitor de la Educación Común. Buenos Aires. Consejo Nacional de Educación, 193-204

Jesinghaus, C. (1924). Sobre la creación de un Instituto Central de Orientación Profesional. Humanidades. Buenos Aires, 385-399

Laufer, R. (1923). Las bases médico-sociales de la orientación profesional. Monitor de la Educación Común. Buenos Aires. Consejo Nacional de Educación, 124-142

Lledó Bercerra, A.I. (2007). La orientación educativa desde la práctica. España: Fundación ECOEM

Lunardi, I. M. (2002). Medicalização, reabilitação, normalização: uma política de educação especial. Trabajo presentado en 25a. Reunião Anual da ANPEd. Caxambu, MG. Recuperado de http://www.anped.org.br/25/marcialiselu nardit15.rtf

Nelson, E. (1920). Aspectos sociales de la educación: El retardado mental y su influencia en el orden social. Monitor de la Educación Común. Buenos Aires. Consejo Nacional de Educación, 156-179

Pacheco, A. L. (2013). La conformación del campo de la orientación educativa siglos XIX y XX en México. Revista Mexicana de Orientación Educativa. [3 época], 10 (25)

Rodriguez Moreno, M. L. (1998). Los fundamentos de la orientación profesional en La Orientación Profesional 1. Teoría. Fundamentos, corrientes, modelos de intervención y fuentes. Barcelona: Ariel Editorial

Santana Vega, L. E. (2003). Orientación educativa e intervención psicopedagógica. Cambian los tiempos, cambian las responsabilidades profesionales. Madrid: Pirámide

Sebastián Ramos, A. (2003). Perspectiva histórica y evolución de la orientación profesional en Orientación Profesional. Un proceso a lo largo de la vida. Madrid: Dikynson Editorial

Southwell, M. (2003). Psicología experimental y Ciencias de la Educación. La Plata: Editorial de la Universidad Nacional de La Plata

Zemelman, H., y Martínez, A. (1987). Funciones del Razonamiento. En Conocimiento y sujetos sociales: Contribución al estudio del presente. México, D.F.: El Colegio de México, 33-62 